\title{
A critical look at connectomics
}

\author{
There is a public perception that connectomics will translate directly into insights for disease. It is essential that \\ scientists and funding institutions avoid misrepresentation and accurately communicate the scope of their work.
}

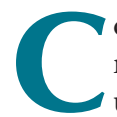

onnectomes are generating interest and excitement, both among neuroscientists and the public. This September, the first grants under the Human Connectome Project, totaling \$40 million over 5 years, were awarded by the US National Institutes of Health $(\mathrm{NIH})$. In the public arena, striking, colorful pictures of human brains have accompanied claims that imply that understanding the complete connectivity of the human brain's billions of neurons by a trillion synapses is not only possible, but that this will also directly translate into insights for neurological and psychiatric disorders. Even a press release from the NIH touted that the Human Connectome Project would map the wiring diagram of the entire living human brain and would link these circuits to the full spectrum of brain function in health and disease. Such grand claims are dangerous because, although a better understanding of brain connectivity is a vital tool for understanding brain function, the immediate gains for therapy from such projects are far from clear. To avoid misrepresentation of connectomics and a potential backlash against the field, it is critical for neuroscientists describing their work as connectomics, their institutions and funding agencies to accurately communicate the scale and scope of their work.

First, the initial grants awarded under the Human Connectome Project do not actually make any claims to offer insights into disease (the subjects are all designated to be healthy adults). Second, it is far from clear that we can expect such a detailed (or useful) wiring diagram of the human brain in the near future. A major source of confusion about the goals of the project stems from the common use of the term 'connectome' to describe investigations of the nervous system that vary by orders of magnitude in their resolution and scope in different animals, including humans, from work investigating connections between brain regions to work investigating connections between individual neurons. Although a broad definition of the word highlights common conceptual themes across otherwise disparate areas of research, there is the unfortunate potential for conflating all of this work in the minds of non-experts, potentially resulting in the misconception that we can soon expect to understand the complete connectivity of the billions of neurons in the human brain. The Human Connectome Project plans to use noninvasive imaging techniques that will allow its investigators to assess the probability that two brain regions are connected. Local connections in brain regions, which are roughly $80 \%$ of the connections in cerebral cortex, are invisible to these imaging methods. Thus, although it will hopefully yield our most complete representation of human brain connectivity to date, the Human Connectome Project will necessarily provide us with partial and probabilistic data. In contrast, connectomics is also used to describe the examination of connectivity at the level of single neurons and synapses. Serial-section electron microscopy was used to map the synaptic connections of the 302 neurons in the nematode C. elegans' nervous system, dubbed the classic connectome because it is currently the only wiring diagram for an animal's entire nervous system at the level of the synapse. Although major neurobiological insights have been made using C. elegans and its known connectivity and genome, there are still many questions remaining that can only be answered by hypothesis-driven experiments. For example, we still don't completely understand the process of axon regeneration, relevant to spinal cord injury in humans, in this comparatively simple system. Thus, a connectome, at any resolution, is only one of several complementary tools necessary to understand nervous system disease and injury.

There are substantial efforts aimed at generating connectomes for other genetically tractable model organisms, including the fruit fly and the mouse, which are partly motivated by the fact that these animals can be used to model human disease. This work has encountered considerable technical and biological challenges that will likely apply to human connectomics projects as well. Animal connectomics experiments generate a large amount of data and how to best use and manage it is still unclear. Even at its comparatively lower resolution, the Human Connectome Project is expected to generate a petabyte of data and successful data management will be a difficult challenge. Animal researchers have also found themselves grappling with an unexpected amount of both inter- and intra-subject variability, even in 'simple' partial connectomes such as the mouse interscutularis neuromuscular junction. It's difficult to imagine the scope of variability that the Human Connectome Project may reveal. Sorting out the differences that matter, particularly those that might be relevant to susceptibility for or pathogenic mechanisms of disease, is far from trivial. Research on connectivity differences in neurological and psychiatric disorders is still controversial and is by no means universally accepted. However, as in the animal studies, this variability will be tremendously interesting in its own right and assessing the scope of human brain variability on the scale offered by the Human Connectome Project will be a major scientific insight.

The Human Genome Project similarly captured both scientists and the public's imagination at its launch, so much so that it became widely expected that personalized medicine would emerge within a few years of the completion of the project. When this didn't happen, the project experienced something of a backlash, asserting that the public had gotten little in return for its investment and ignoring basic scientific insights. It's tempting to sell the Human Connectome Project, and connectomics in general, as directly relevant to disease, particularly given the public money invested. However, given the challenges that this field is facing, it seems ill-advised to present connectomics as providing immediate answers for disease when it is clear that this is a long-term goal that will require the continued support and collaboration of the neuroscience community and the tax-paying public. 